\title{
Composites of Enteric Polymer/Magnetite: Preparation and Application in Release Processes
}

\author{
S.V. de Lima and H.P. de Oliveira*
}

Colegiado de Pós-Graduação em Ciência dos Materiais, Universidade Federal do Vale do São Francisco, 48902-300, Juazeiro, BA, Brazil

\begin{abstract}
The controlled release of drugs represents an interesting procedure applied in the minimization of side effects and in the therapeutic action of pharmacological molecules. With the aim of measuring the kinetics of release of aspirin dispersed in composite of an enteric polymer (Eudragit L-100) in the presence/absence of magnetic particles, we have considered the measurement of fluorescence as a convenient tool. The results indicate that rate of release and amount of dispersed drug in solution varies directly with the concentration of magnetite in the composite, as a consequence of strong interaction between components of microparticles.
\end{abstract}

Keywords: Controlled release, magnetite, fluorescence.

\section{INTRODUCTION}

Aspirin is a non-steroidal anti-inflammatory drug with analgesic properties [1], applied in the secondary prevention of atherosclerosis [2, 3]. The modification in human blood induced by aspirin represents other important application, which is based on blood fluidization promoted by inhibition of platelet aggregation [4].

The optimal dose of aspirin depends on the clinical conditions of each patient, if considering the risk of side effects (as a consequence of continued administration of this drug) such as renal function deterioration. As a consequence, the control of ultra-low doses of aspirin (adequate to prevention of rheumatoid arthritis) [5] and its controlled release [1] contribute with the minimization in the side effects such as ulcer, hemorrhage and stomach diseases [6-9] and to maximize the expected results.

In this work, we have used the emission properties of aspirin in alkaline media, in order to quantify the amount of dispersed drug released in solution after encapsulation by enteric block copolymers. The encapsulation of aspirin in an enteric polymer and subsequent release in solution is controlled by swelling/ solubilization of drug in the polymeric matrix, whose solubility depends on the $\mathrm{pH}$ of media. On the other side, magnetic-polymeric composites have been progressively used in applications such as drug target and drug release, due to their biocompatibility and biodegradability [10]. The interaction with drug results

*Address corresponding to this author at the Colegiado de Pós-Graduação em Ciência dos Materiais, Universidade Federal do Vale do São Francisco, 48902300, Juazeiro, BA, Brazil; Tel: +55-74-2012.7644; Fax: +55-74-2102.7645; E-mail: helinando.oliveira@univasf.edu.br in the enhancement of stability against oxidation [11] and subsequent application of polymeric structures as a shell [12]. Magnetic particles have been widely reported as drug carriers, $[13,14]$ with the release process induced by a given magnetic field. In the mixed system (drug/ magnetite/ enteric polymer), the control of release process is provided by the use of Eudragit L100 , a polymeric matrix composed by a hydrophilic monomeric unit (metacrylic acid) and a hydrophobic part (methyl metacrylate). This process is dominated by the ionization degree of carboxylic group, which depends on the $\mathrm{pH}$ of media and affects the polymeric conformation $[15,16]$

Based on these properties, the kinetics of drug release is controlled from the introduction of variable amount of magnetite in the composite and by $\mathrm{pH}$ of media. The fluorescence of aspirin was measured in order to identify the concentration of released drug in solution.

\section{MATERIALS AND METHOD}

\subsection{Materials}

Aspirin, methanol, ferric chloride $\left(\mathrm{FeCl}_{3}\right)$, ferrous sulphate $\left(\mathrm{FeSO}_{4}\right)$ and ammonium hydroxide $\left(\mathrm{NH}_{4} \mathrm{OH}\right)$ were acquired from Sigma-Aldrich (USA). Eudragit L100 was kindly donated from Röhm Pharma (Germany) and all of reagents were used as received.

We have used the standard procedure of coprecipitation to produce $\mathrm{Fe}_{3} \mathrm{O}_{4}$ nanoparticles $[17,18]$ from reaction between $\mathrm{Fe}^{3+}$ and $\mathrm{Fe}^{2+}$ in a ratio of $2: 1$ in the presence of $\mathrm{NH}_{4} \mathrm{OH}$. Aqueous solution of $0.1 \mathrm{M}$ $\mathrm{FeCl}_{3}(30 \mathrm{~mL})$ and $0.1 \mathrm{M} \mathrm{FeSO}_{4}(15 \mathrm{~mL})$ were mixed, after that $3 \mathrm{~mL}$ of $6.5 \mathrm{M} \mathrm{NH}_{4} \mathrm{OH}$ was dropwised in the mixture under intense stirring. The peptization of 


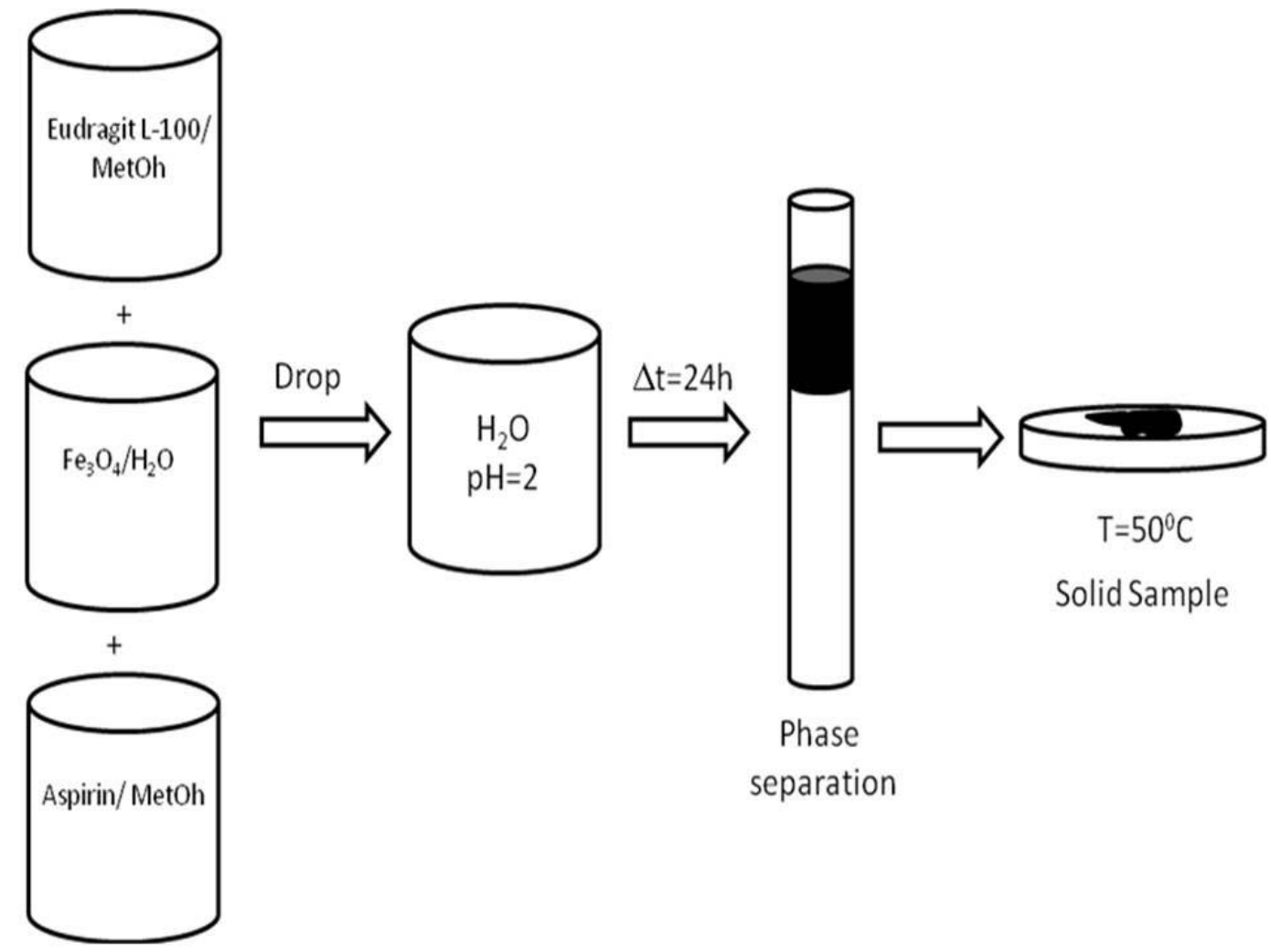

Figure 1: Scheme of encapsulation of mixed particles of magnetite/ aspirin.

synthesized particles was provided by chloride, which was eliminated from solution after several washing procedures. The magnetic separation of particles from solution and substitution of residual solution by deionized water was repeated until the level of resistivity of residual solution reach the required level (18.2 $\mathrm{M} \Omega \mathrm{cm})$. After complete evaporation of water, a powder of $300 \mathrm{mg}$ of magnetite was obtained, from which aliquots were used for preparation of composites.

The encapsulation was obtained from the solubilization of aspirin and Eudragit L-100 in methanol (as indicated in the Figure 1). In this situation, $200 \mathrm{mg}$ of Eudragit L-100 and $250 \mathrm{mg}$ of aspirin were dissolved in $20 \mathrm{~mL}$ of methanol, separately. After this step, these solutions were mixed and the final product receipt 10 $\mathrm{mL}$ of magnetic fluid at different concentrations (corresponding mass of magnetite of $0.0,0.5,0.7$ and $1.1 \mathrm{mg}$ ) according Table 1. Finally, the ternary system was dropped in acidified aqueous solution $(0.5 \mathrm{M}$ of $\mathrm{HCl}(\mathrm{pH}=2))$ under constant stirring after that the encapsulation of aspirin and magnetite takes place, with subsequent precipitation of aggregates in aqueous solution.

The complete separation of solid phase from aqueous solution was obtained after 24 hours with elimination of residual water/methanol under heating condition at a fixed temperature of $60^{\circ} \mathrm{C}$. The dried nanocomposite with mass in order of $405 \mathrm{mg}$ was obtained and applied during release process.

Table 1: Characteristic Time, Concentration of Drug at Different Concentration of Encapsulated Magnetite Particles and Constants

\begin{tabular}{|c|c|c|c|c|c|}
\hline Sample & $\begin{array}{c}\mathrm{Fe}_{3} \mathrm{O}_{4} \text { concentration } \\
(\mathbf{m g} / \mathbf{m L})\end{array}$ & $\begin{array}{c}\text { Characteristic time of } \\
\text { drug release }(\mathbf{m i n})\end{array}$ & $\begin{array}{c}\text { Concentration of } \\
\text { released drug }(\boldsymbol{\mu} \mathbf{g} / \mathbf{m L}) \\
- \text { Saturation } \text { condition }\end{array}$ & A (a.u.) - Eq. 1 B (a.u.) - Eq. 1 \\
\hline \hline Aspirin & 0.00 & 27.29 & 2.14 & -63821.97 & 72371.74 \\
\hline C3 & 0.05 & 17.25 & 3.61 & -99284.09 & 116251.53 \\
\hline C2 & 0.07 & 16.44 & 4.15 & -114298.59 & 132044.31 \\
\hline C1 & 0.11 & 12.83 & 3.91 & -98981.58 & 131060.96 \\
\hline
\end{tabular}


The experiments of drug release were established from introduction of tablets of composites (mass of 405 $\mathrm{mg}$ ) in $1 \mathrm{~L}$ of water at neutral $\mathrm{pH}$ under constant stirring. The amount of released aspirin was determined from the measurement of intensity of emission peak at $400 \mathrm{~nm}$ [19] by the use of aliquots (4 $\mathrm{mL}$ ) of solution during the release (interval of time between 0 and 40 minutes) with excitation at $344 \mathrm{~nm}$. The complete dispersion of aspirin in water was obtained from the insertion of $\mathrm{NaOH}(1 \mathrm{M})$ in the solution under test.

\subsection{Apparatus}

Luminescence properties were studied by the use of a PC1 spectrofluorimeter (ISS, USA) at $(25.0 \pm 0.1)^{\circ} \mathrm{C}$. The samples were placed in a $5 \mathrm{~mL}$ cuvette with a path length of $1.0 \mathrm{~cm}$ and excited at $344 \mathrm{~nm}$, while the emission was recorded from 350 to $550 \mathrm{~nm}$. The infrared spectra were analyzed from the use of a Shimadzu IR Prestige 21 (Shimadzu, Japan) by the use of compressed pellets of $\mathrm{KBr}$ while the images were obtained from the use of a SEM Hitachi model TM1000 (Hitachi, Japan). All of experiments were repeated three times.

\section{RESULTS AND DISCUSSION}

The concentration of released aspirin in the solution was determined from a calibration curve obtained in the complete absence of other components (magnetite and Eudragit L-100). With increasing concentration of aspirin (in the range of $0.25 \mathrm{mg} / \mathrm{mL}$ to $0.97 \mu \mathrm{g} / \mathrm{mL}$ ) the emission was measured (as shown in the Figure 2). As

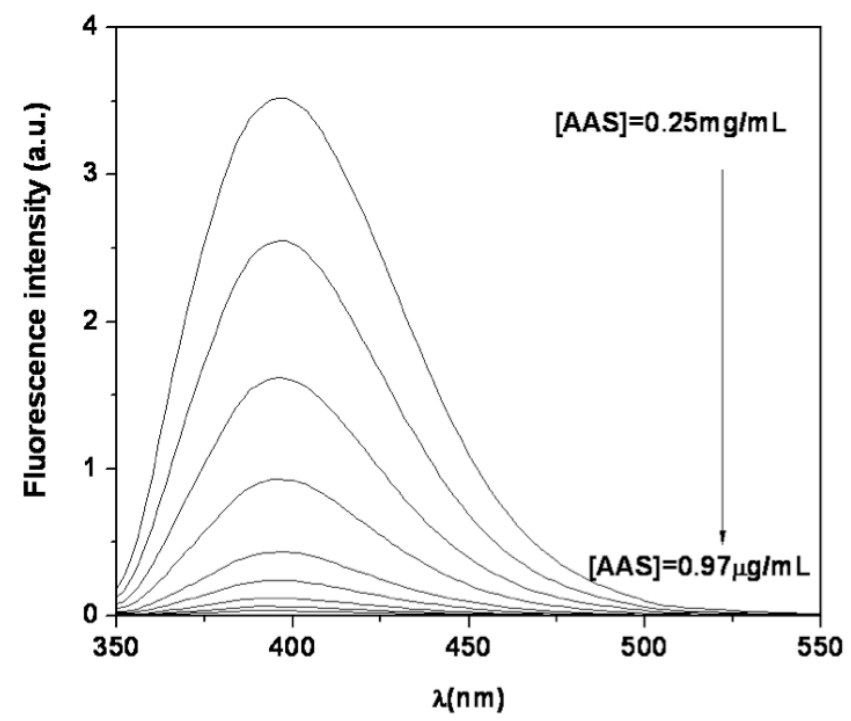

Figure 2: Emission of aqueous solution of aspirin as a function of concentration. we can see, the whole emission increases with the concentration, in an indication that peak at $400 \mathrm{~nm}$ can be used as a convenient parameter in the measurement of amount of aspirin dispersed in solution. The plot of maximum of fluorescence intensity of aspirin versus concentration is shown in the Figure 3. By the use of a calibration curve (with data described in the inset of Figure 3), it is possible to correlate the fluorescence of solution with the corresponding released amount of aspirin.

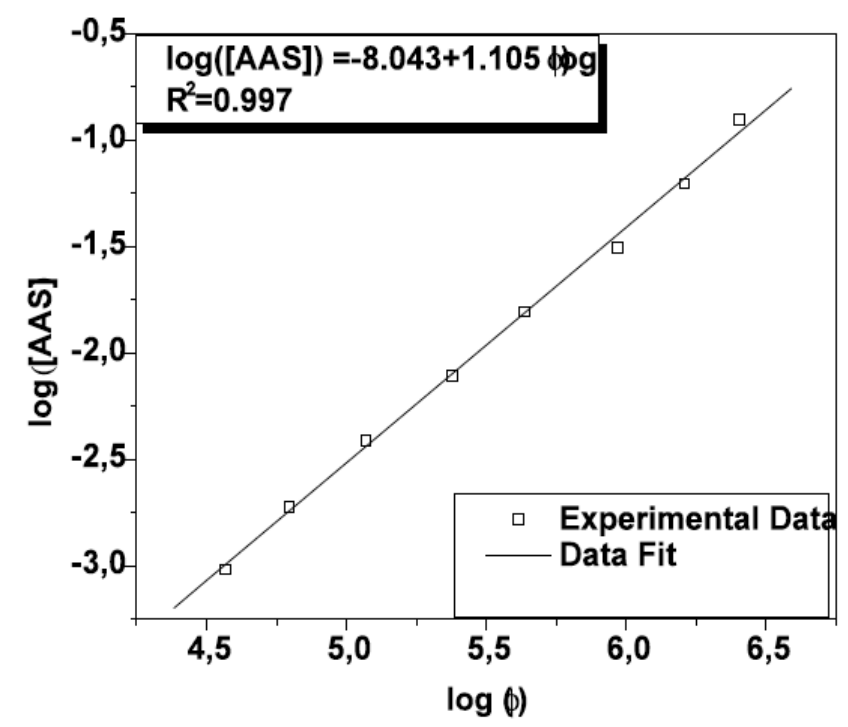

Figure 3: Dependence of emission peak of aspirin $(400 \mathrm{~nm})$ with concentration.

If considering the properties of Eudragit L-100, the encapsulation of aspirin is established at low $\mathrm{pH}$ (minimal solubility of enteric polymer). After the encapsulation, described in the previous section, we observed the formation of spherical structures in scale of micrometers.

In the complete absence of magnetic particles, the particles are symmetrical, as we can see in the microscopy of Figure 4. However, the introduction of magnetite promotes the deformation of particles with elongated shape, as shown in the Figure 5, with identification of metal microparticles dispersed in the core of particles.

The images obtained for composite containing enteric polymer, magnetite and aspirin indicate the same behavior obtained for composites containing magnetite, with the increase in the number of elongated structures and distribution of aggregates into and between particles, as we can see in the Figure $\mathbf{6}$, indicating that encapsulation efficiency is typically reduced in the ternary system. 


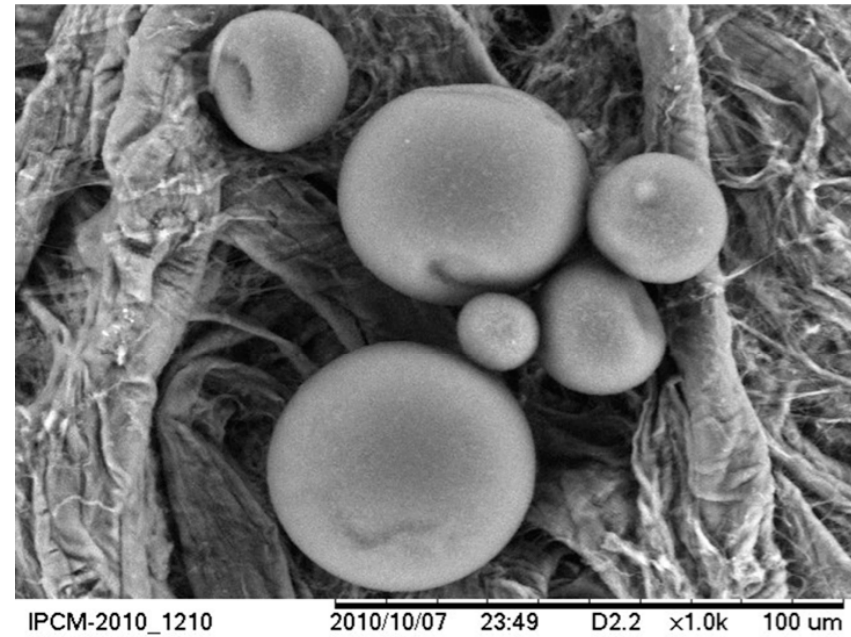

Figure 4: SEM image of microparticles of Eudragit L-100.

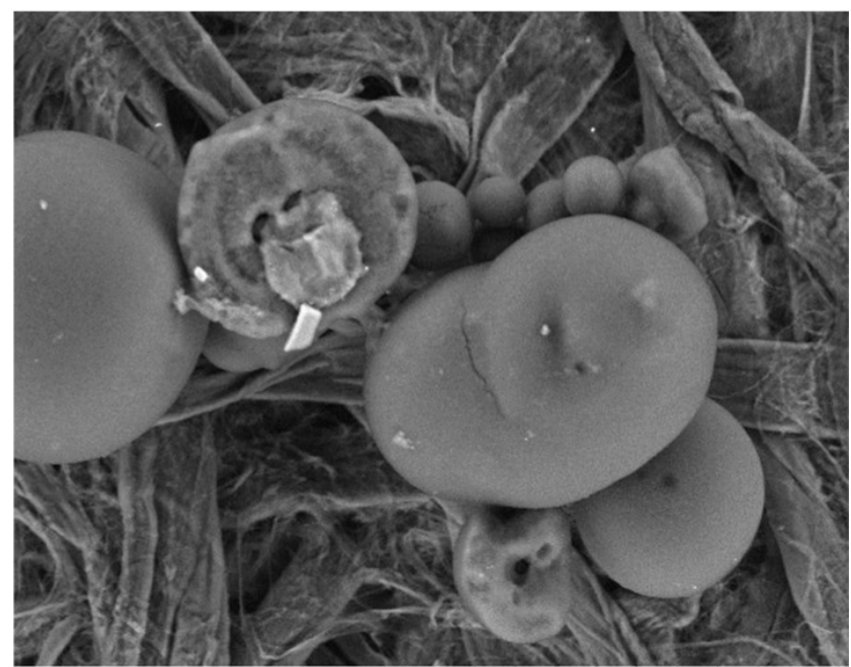

Figure 5: SEM image of microparticles of Eudragit L-100 and magnetite.

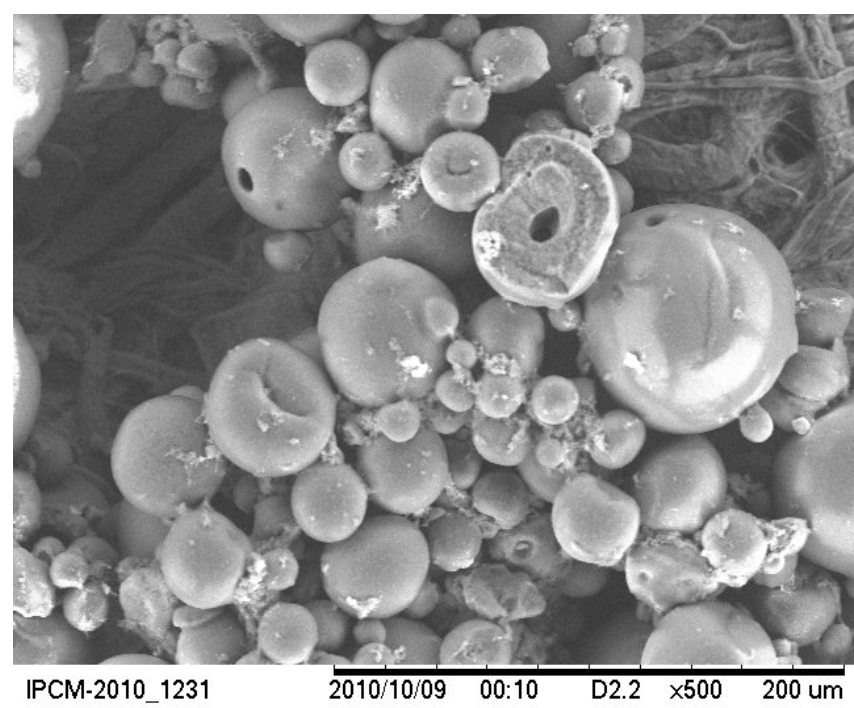

Figure 6: SEM image of microparticles of Eudragit L-100, aspirin and magnetite.
The characterization of solid samples was provided from the measurement of FTIR spectra, as shown in Figure 7. The peak at $580 \mathrm{~cm}^{-1}$ characterizes the $\mathrm{Fe}-\mathrm{O}$ group to the magnetite while peaks at 1750 and 3300 $2500 \mathrm{~cm}^{-1}$ characterizes the $\mathrm{C}=\mathrm{O}$ (ester) and $\mathrm{O}-\mathrm{H}$ carboxylic acid groups, respectively, indicating the presence of aspirin in the composite.

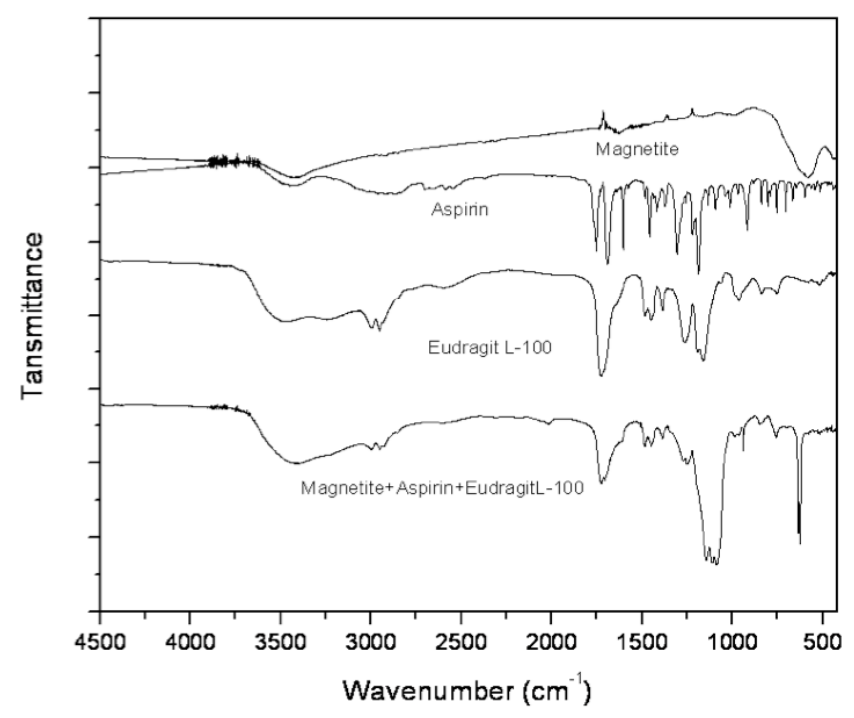

Figure 7: FTIR spectra of components (magnetite, aspirin and Eudragit L-100) and nanocomposite.

The FTIR of Eudragit L-100 is equivalent to the reported in the Refs. [20-22]. As we can see, the peaks at $3500,3000,1736,1195$ and $1165 \mathrm{~cm}^{-1}$ are attributed to the free form of carboxylic acid, $\mathrm{CH}_{\mathrm{x}}$ vibration, the dimer of carboxylic ester, and $\mathrm{C}-\mathrm{O}$ bonds of carboxylic ester and acid, respectively. In the case of ternary system, it is possible to verify an additional peak at 636 $\mathrm{cm}^{-1}$ which characterizes the presence of $\mathrm{Fe}-\mathrm{O}-\mathrm{Fe}$.

With the previous characterization of components of system, we have analyzed the release process of aspirin as a function of concentration of magnetite dispersed in composites. The results (as shown in the Figure 8) indicate that release of drug is described as a function of type:

Intensity $=A e^{-t / \tau}+B$

where Intensity represents the fluorescence peak measured at $400 \mathrm{~nm}, A$ and $B$ are constants, $t$ is the release time and $\tau$ is the characteristic release time (parameters for different concentration of magnetite are summarized in the Table 1). Note that $A$ assumes negative value while $B$ is positive for all of samples. At $\mathrm{t}=0$, (Intensity $=\mathrm{B}+\mathrm{A}$ ) represents the fluorescence of residual aspirine (non-encapsulated molecules) while 
at elevated values of time, the intensity tends to $B$. Similarly from definition established during charge/ discharge of capacitor, characteristic time defines the required time for $63 \%$ of release of total amount of drug in each system.

As we can see, the increase in the concentration of magnetite dispersed in solution is accomplished by reduction in the encapsulation efficiency, as detected in the level of fluorescence measured at $t=0$ (see Figure 8), in an indication that magnetite aggregates minimize the encapsulation efficiency of aspirin. It is a consequence of deformation and rupture induced by magnetite in the capsules of Eudragit L-100, resulting in an increase in the concentration of non-encapsulated aspirin.

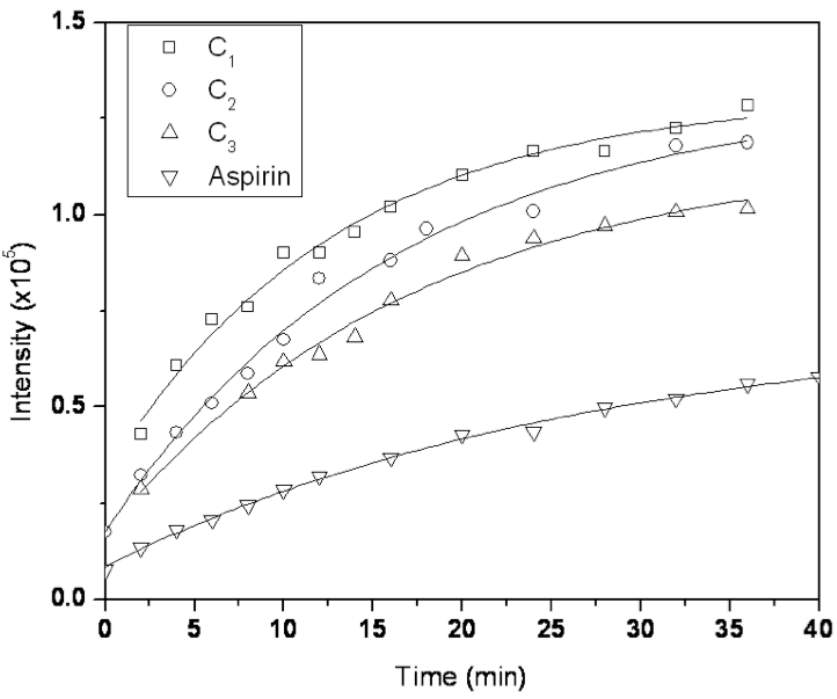

Figure 8: Release profile of aspirin encapsulated with Eudragit L-100 at different concentration of magnetite.

However, the amount of magnetite dispersed in composite affects the kinetics of release and the amount of released drug in water. As we can see in Table 1, the inclusion of $1.1 \mathrm{mg}$ of magnetite in the composite promotes the reduction in the characteristic time of release from $27.29 \mathrm{~min}$ (absence of magnetic fluid) to $12.83 \mathrm{~min}$, in an indication that strong interaction between magnetite and enteric polymer is established with the control in the porosity and solubility of synthesized particles in water. At the same time in which the characteristic time of release is affected by inclusion of magnetite, it is possible to verify that progressive inclusion of magnetite in the composite affects the amount of released drug, as clearly indicated in the Table 1 . These results act as a strong indication that magnetite contributes with the control in the kinetics of release of encapsulated drug in the absence of magnetic field. The influence of an external field on the response of composites represents the next step of this study.

\section{CONCLUSIONS}

The fluorescence of aspirin represents an important parameter in the detection of released drug from composites of enteric polymer in aqueous solution. The introduction of magnetic particles into the core of enteric polymer aggregates affects the kinetics of release, from the elongation of synthesized microparticles in an association with the increase in the released drug rate; a direct consequence of size and porosity control of dispersed microparticles in aqueous solution, induced by aggregation of magnetite and aspirin. The results indicate that magnetite act as an accelerating component with strong contribution on the release rate of drug.

\section{ACKNOWLEDGEMENTS}

The authors wish to thank CNPq, FAPESB, FACEPE, CAPES and FINEP.

\section{REFERENCES}

[1] Zhang SF, Feng YK, Zhang L, Guo JT, Xu YS Biodegradable Polyesterurethane Networks for Controlled Release of Aspirin. J Appl Polym Sci 2010; 116: 816-7.

[2] Wada M, Nishiwaki J, Yamane T, Ohwaki Y, Aboul-Enein HY, Nakashima K. Interaction study of aspirin or clopidogrel on pharmacokinetics of donepezil hydrochloride in rats by HPLC-fluorescence detection. Biomed Chromatogr 2007; 21 : 616-20. http://dx.doi.org/10.1002/bmc.794

[3] Byun HS, Youn YN, Yun YH, Yoon SD. Selective separation of aspirin using molecularly imprinted polymers. Sep Purif Technol 2010; 74: 144-53 http://dx.doi.org/10.1016/j.seppur.2010.05.017

[4] Curini R, D'Ascenzo G, Fraioli A, Gentili A, Girelli G, Orru MA, Materazzi S. Analusis 1999; 27: 786-94 http://dx.doi.org/10.1051/analusis:1999145

[5] Shadick NA, Karlson EW, Cook NR, Maher NE, Buring JE, Lee IM. Low-Dose Aspirin in the Primary Prevention of Rheumatoid Arthritis: The Women's Health Study. Arthitris Care Res 2010; 62: 545-50. http://dx.doi.org/10.1002/acr.20042

[6] Hawkey CJ. Nonsteroidal anti-inflammatory drug gastropathy. Gastroenterology 2000; 119: 521-35. http://dx.doi.org/10.1053/gast.2000.9561

[7] Allison MC, Howatson AG, Torrance CJ, Lee FD, Russell RI Gastrointestinal Damage Associated with the Use of Nonsteroidal Antiinflammatory Drugs. N Engl J Med 1992; 327: 749-54.

http://dx.doi.org/10.1056/NEJM199209103271101

[8] Wolfe MM, Lichtenstein DR, Singh G. Gastrointestinal toxicity of nonsteroidal antiinflammatory drugs. N Engl J Med 1999; 340: 1888-99.

http://dx.doi.org/10.1056/NEJM199906173402407 
[9] Rich M, Scheiman JM. Nonsteroidal anti-inflammatory drug gastropathy at the new millennium: Mechanisms and prevention. Semin Arthritis Rheum 2000; 30: 167-79. http://dx.doi.org/10.1053/sarh.2000.16643

[10] Zeng P, Kline TL, Wang JP, Wiedmann TS. Thermal response of superparamagnetic particles suspended in liquid and solid media. J Magn Magn Mater 2009; 321: 373-6. http://dx.doi.org/10.1016/j.jmmm.2008.09.032

[11] Zhou LL, Yuan JY, Yuan WZ, Sui XF, Wu SZ, Li ZL, et al. Synthesis, characterization, and controllable drug release of $\mathrm{pH}$-sensitive hybrid magnetic nanoparticles. J Magn Magn Mater 2009; 321: 2799-804. http://dx.doi.org/10.1016/j.jmmm.2009.04.020

[12] Zavisova V, Koneracka $M$, Muckova $M$, Kopcansky $P$, Tomasovicova N, Lancz G, et al. Synthesis and characterization of polymeric nanospheres loaded with the anticancer drug paclitaxel and magnetic particles. J Magn Magn Mater 2009; 321: 1613-36. http://dx.doi.org/10.1016/j.jmmm.2009.02.097

[13] Liu TY, Hu SH, Liu KH, Liu DM, Chen SY. Preparation and characterization of smart magnetic hydrogels and its use for drug release. J Magn Magn Mater 2006; 304: E397-9. http://dx.doi.org/10.1016/j.jmmm.2006.01.203

[14] Huang LY, Yang MC. Behaviors of controlled drug release of magnetic-gelatin hydrogel coated stainless steel for drugeluting-stents application. J Magn Magn Mater 2007; 310: 2874-6.

http://dx.doi.org/10.1016/j.jmmm.2006.11.151

[15] de Oliveira HP, Tavares GF, Nogueiras C, Rieumont J. Physico-chemical analysis of metronidazole encapsulation processes in Eudragit copolymers and their blending with amphiphilic block copolymers. Int J Pharm 2009; 380: 55-61. http://dx.doi.org/10.1016/j.ijpharm.2009.06.028
[16] de Oliveira HP, Albuquerque JJF, Nogueiras C, Rieumont J. Physical chemistry behavior of enteric polymer in drug release systems. Int J Pharm 2009; 366: 185-9. http://dx.doi.org/10.1016/j.ijpharm.2008.08.041

[17] Sun J, Zhou SB, Hou P, Yang Y, Weng J, Li XH, et al. Synthesis and characterization of biocompatible $\mathrm{Fe}_{3} \mathrm{O}_{4}$ nanoparticles. J Biomed Mater Res 2007; 80A: 333-41. http://dx.doi.org/10.1002/jbm.a.30909

[18] Jain KB, Morales MA, Sahoo SK, Leislie-Pelecky DL, Labhasetwar V. Iron oxide nanoparticles for sustained delivery of anticancer agents. Mol Pharmaceutics 2005; 2: 194-205. http://dx.doi.org/10.1021/mp0500014

[19] Venema DP, Hollaman Peter CH, Janssen Karin PLTM, Katan MB. Determination of acetylsalicylic acid and salicylic acid in foods, using HPLC with fluorescence detection. J Agric Food Chem 1996; 44: 1762-1767. http://dx.doi.org/10.1021/j950458y

[20] Lin SY, Liao CM, Hsiue GH. A Reflectance Ftir/Dsc Microspectroscopic Study of the Nonisothermal Kinetics of Anhydride Formation in Eudragit L-100 Films. Polym Degrad Stab 1995; 47: 299-303. http://dx.doi.org/10.1016/0141-3910(94)00129-V

[21] Zaroni M, Ramos DT, Murakami FS, Carvalho MAS, Janissek PR, Andreazza IF, et al. Thermal behavior and interaction studies of theophylline with various excipients. Lat Am J Pharm 2008; 27: 191-6.

[22] Gonzalez M, Galano A, Rieumont J, Lopez T, Dupeyron D, Albaran L. Drug-Matrix Interactions in Nanostructured Materials Containing Acetyl Salicylic Acid Using an Enteric Polymer As a Coating. J Phys Chem C 2008; 112: 20222-6. http://dx.doi.org/10.1021/jp806572g 